\title{
MiRNA-182-5p aggravates experimental ulcerative colitis via sponging Claudin-2
}

\author{
Siwen Tang ${ }^{1} \cdot$ Wentao Guo ${ }^{1} \cdot$ Liumin Kang $^{2} \cdot$ Jinghua Liang $^{1}$ (])
}

Received: 1 March 2021 / Accepted: 14 September 2021 / Published online: 8 October 2021

(c) The Author(s) 2021

\begin{abstract}
Tight junction proteins play crucial roles in maintaining the integrity of intestinal mucosal barrier. MiRNA-182-5p is capable of targeting claudin- 2 which is one of the vital tight junction proteins and the effect and mechanism of miRNA-182-5p was explored here in the DSS-induced colitis model. The pathological conditions were evaluated via hematoxylin and eosin staining. The gene expression level was assessed via PCR. Quantitative immunohistochemistry analysis was performed for the measurement of claudin-2. microRNA.org online tool was used for target gene prediction. Luciferase reporter assay and RNA pull-down assay were performed to detect the target of miRNA-182-5p. The inflammatory and oxidative stress level were measured using corresponding kits. MiRNA-182-5p was highly expressed in colitis model and miRNA-182-5p inhibitor exerted protective effects on colitis induced by DSS in mice. The protective effects includded improvement of pathological changes, increases in anti-inflammation and anti-oxidative genes, and up-regulation of TGF- $\beta 1$. Claudin- 2 mRNA was predicted as the target of miRNA-182-5p, which was validated via luciferase reporter assay and RNA pull-down assay. Claudin-2 overexpression was found in miRNA-182-5p inhibitor group. Consistent with the role of miRNA-182-5p, claudin-2 overexpression also exerted protective effects on DSS-induced colitis in mice. Inhibition of miRNA-182-5p exerted protective effects on colitis via targeting and upregulating claudin-2. The findings in study provide a new therapeutic strategy for colitis treatment and lay the foundation for future study.
\end{abstract}

Keywords MiRNA-182-5p $\cdot$ Claudin-2 $\cdot$ Colitis $\cdot$ Inflammation $\cdot$ Intestinal mucosal barrier $\cdot$ Anti-oxidative

\section{Introduction}

Inflammatory bowel disease is a refractory, nonspecific, debilitating inflammatory disease [1]. Ulcerative colitis and Crohn's disease are two major forms of inflammatory bowel disease [2]. Common clinical manifestations include abdominal pain, fever, diarrhea, hematochezia, tenesmus, vomiting, and an increase in frequency of defecation [3, 4]. The incidence of inflammatory bowel disease is soaring globally [5-7]. In addition, chronic colorectal inflammation has been confirmed as the vital contributor of tumorigenesis [8]. Nowadays, the known causes of inflammatory bowel

Jinghua Liang

tsw123tsw@126.com

1 Shenzhen Traditional Chinese Medicine Anorectal Hospital (FuTian), No. 1 Songling Road, Futian District, 518000 Shenzhen, China

2 Suzhou Science and Technology City Hospital Affiliated to Nanjing Medical University, 215000 Suzhou, China disease includes environmental factors, genetic factors and immunity factors [9]. The exact pathogenesis is complex and yet to be further elaborated. The current treatment such as immunomodulators and antibiotic can only achieve clinical remission [10]. Meanwhile, there is individual difference in patients with inflammatory bowel disease, which has made the treatment more difficult. Therefore, it's urgent to elaborate the vital regulatory mechanism without individual difference and develop effective therapeutic drugs based on it.

MicroRNAs (miRNAs), as non-coding RNAs, function by inhibiting the translation or promoting the degradation of mRNA, which are powerful gene regulators of gene expression at transcriptional and post-transcriptional levels. miRNAs have been involved in a lot of cell activities including metabolism, cell proliferation and differentiation, cell cycle, aging, immune responses, inflammation and so forth, which are also served as the vital biomarkers and therapeutic targets in many diseases [12-15]. MiRNA-219a-5p is found to have inhibitory effects on intestinal inflammation via inhibition of immune responses mediated by $\mathrm{T}$ helper (Th)1/Th17, 
which may become a promising therapeutic target for colitis [16]. MiRNA-125a inhibits the inflammation of intestinal mucosal via targeting ETS-1 in patients [17]. Therefore, it is beneficial to screen for? the vital miRNA that is crucial in the onset and progress of colitis.

Increasing evidence has shown that intestinal mucosal barrier defects are the core factors for IBD [18]. When the intestinal mucosal barrier is impaired, the intestinal lamina propria will be invaded by toxins and pathogenic bacteria $[19,20]$. The intestinal mucosal barrier is mainly maintained by the tight junction proteins which consist of claudins, zonula occluden-1 and occluding. Claudin- 2 as one of the tight junction proteins is downregulated in inflammatory bowel disease, which contributes to the further progress of IBD [21]. Reducing the decrease of Claudin-2 may be a good therapeutic strategy for IBD. It is promising to find the vital miRNA that is capable of regulating Claudin-2 expression. After bioinformatics analysis (microRNA.org online tool), Claudin-2 is predicted to be the target gene of miRNA182-5p and the effects of miRNA-182-5p on inflammatory bowel disease remains unknown. Herein, we are the first to investigate the effects of miRNA-182-5p and its association with Claudin-2 in dextran sulphate sodium (DSS) induced colitis.

\section{Materials and methods}

\section{Experimental animals}

The C57BL/6 mice (7-8 week, $\mathrm{n}=80$ ) from Cancer prevention and treatment center of Sun Yat sen University (SYXK(yue)2021-0255) were raised under specific pathogen free conditions with humidity of $65 \%$ at $22{ }^{\circ} \mathrm{C}$ in the experimental animal center. This study is approved by committee of Shenzhen Traditional Chinese Medicine Anorectal Hospital and the whole animal experimental processes were performed in line with the Committee guidelines of animal research.

\section{The establishment of experimental colitis model and treatment [22]}

After getting acclimatized to the environment, the mice (ten mice per group) were randomly divided into control group, model group, negative control (NC) group and inhibitor group.

The mice in the model group were treated with 4\% DSS (MP Biomedicals, Santa Ana, California, USA) for one week. The mice in control group were treated with equal volume of sterilized water. For NC group, the mice after establishment of experimental colitis were treated with miRNA-182-5p negative control (200 $\mu$ l, Sangon Biotech
(Shanghai) Co., Ltd. Shanghai, China) via intravenous injection. For inhibitor group, DSS mice were treated with miRNA-182-5p (200 $\mu$ l, Sangon Biotech) via intravenous injection. For Claudin-2 NC group, the mice after establishment of experimental colitis were treated with negative control of plasmid ( $200 \mu \mathrm{l}$, Sangon Biotech) via intravenous injection. For Claudin-2 group, the mice after establishment of experimental colitis were treated with Claudin-2 overexpression plasmid ( $200 \mu \mathrm{l}$, Sangon Biotech) via intravenous injection. During the period, the stool consistency, body weight and intestinal bleeding were monitored every day.

\section{Hematoxylin and eosin staining}

After treatment, the mice in the study groups were sacrificed via euthanasia. The colonic tissues were dissected, isolated and washed with cold PBS. Then the samples were fixed with $4 \%$ paraformaldehyde. Embedded in paraffin, the samples were sliced into $5 \mu \mathrm{m}$ sections. Then the deparaffinization was performed using xylene and the samples were rehydrated with gradient elution. Subsequently, the hematoxylin was added to stain the sections for ten minutes and eosin for one minute. After dehydration, the sections were vitrificated and examined using microscope (Olympus Corporation, Japan).

\section{Immunohistochemical analysis}

After treatment, the mice in the study groups were sacrificed via euthanasia. The colonic tissues were dissected, isolated and washed with cold PBS. Then the samples were fixed with $4 \%$ paraformaldehyde. Embedded in paraffin, the samples were sliced into $5 \mu \mathrm{m}$ sections. The sections were heated in the PBS in a microwave. After cooling down, the serum blocking solution was added to block the sections for half an hour at $37^{\circ} \mathrm{C}$. Then the sections were incubated with antiClaudin-2 antibody(1:1000, \#ab53032, Abcam, England) at $4{ }^{\circ} \mathrm{C}$ overnight and secondary antibody (\#ab205718, Abcam) at $37^{\circ} \mathrm{C}$ for one hour. After being washed with PBS, the sections were stained with diaminobenzidine for five minutes and hematoxylin for two minutes. Finally, the sections were observed under the microscope (Olympus Corporation).

\section{Real-time qRT-PCR assay (quantitative real-time PCR qRT-PCR)}

TRIzol reagent (Thermo Fisher Scientifc, Inc., Waltham, MA, USA.) was used for istolating total RNAs from colonic tissues according to the protocol of manufacturer. MiScript II RT Kit (218161, Qiagen, Germany) was used for the reverse transcription from miRNA to cDNA. The PCR assay were performed on RT-qPCR detection system (Applied Biosystems; Thermo Fisher Scientific, Inc., U.S.A.). The 
amplification conditions and miR-182-5p primers were the same with the previous study [23]. The primers were obtained from Sangon Biotech for Claudin-2 F:5'-TTCATC GGCAACAGCATCG-3' (Forward) and R:5'-GGTTATAGA AGTCCCGGATGA-3' (reverse);

\section{Assessment of colonic inflammation and damage}

The colonic tissues were homogenized and submitted for centrifugation $(20000 \times \mathrm{g}, 25 \mathrm{~min})$. The supernatants were collected for detection. The inflammatory factors including transforming growth factor (TGF)- $\beta$, interleukin (IL)21 , IL-17, IL-6 in the supernatants were assessed herein via using the corresponding ELISA kits (eBioscience) according to the manufacturer's protocols, including TGF$\beta 1$ (Transforming Growth Factor Beta 1) ELISA Kit (Cat: E-EL-0162c; Elabscience Biotechnology Co., Ltd, Wuhan, China), Mouse Interleukin-21 ELISA Kit (Cat: ZN2661; Beijing baiaolaibo Technology Co., Ltd, Beijing, China), Mouse Interleukin 17,IL-17 ELISA Kit (Cat: E-EL-M0047c; Elabscience Biotechnology) and Mouse IL-6(Interleukin 6) ELISA Kit (Cat: E-EL-M0044c; Elabscience Biotechnology). The colonic damage index was evaluated according to the previous report [24]. The colonic damage index was scored on a scale of $0-5$. The entire colon of each animal was rinsed with cold physiological saline to remove feces. The colon damage was examined visually and immediately. The colonic damage was scored as 0 , for no damage; 1 , for hyperemia and no ulcers; 2 , for linear ulcer with no significant inflammation; 3 , for Linear ulcer with inflammation at one site; 4 , for two or more sites of ulceration or inflammation and the area extending $<1 \mathrm{~cm} ; 5$, for two or more major sites of ulceration or inflammation extending $>1 \mathrm{~cm}$.

\section{Assessment of MDA and SOD level}

After treatment, the mice in the study groups were sacrificed via euthanasia. The colonic tissues were dissected. The malondialdehyde (MDA) in the supernatants were detected as described before [25]. The superoxide dismutase (SOD) level in the supernatants was detected using Superoxide Dismutase Activity Assay Kit (Cat: E-BC-K020-M; Elabscience Biotechnology). The detection of SOD was performed according to the manufacturer's protocols.

\section{Luciferase reporter assay [26]}

Claudin-2 is predicted as the target of miRNA-182-5p through microRNA.org online tool (http://microRNA.org). The 3'UTR sequence of claudin-2 (WT) or its mutated type (MUT) was cloned into the psiCHECK2 vector (Invitrogen). The miR-182-5p or miR-182-5p mimic with mutant or wild-type of claudin-2 were co-transfected into HEK293 cells using Lipofectamine 2000 (Thermo Fisher Scientifc) in accordance with the protocols of manufacturer. After transfection for $48 \mathrm{~h}$, luciferase activity was detected via using dual luciferase reporter assay system (Promega Corporation, Madison, USA).

\section{RNA pull-down assay [27]}

The normal intestinal epithelial cell line Caco-2 was purchased from iCell Biotechnology Co., Ltd (Shanghai, China). The cells were cultured in RPBM 1640 medium supplemented with $10 \%$ fetal calf serum and maintained at $37{ }^{\circ} \mathrm{C}$ with $5 \% \mathrm{CO}_{2}$ in a humidified atmosphere. The RNA pull-down assay was performed via using Magna RIP Kit (Millipore (China) Co., Ltd, Shanghai, China). The cells were transfected with bio-wild-miRNA-182-5p (miRNAbio), bio-mutated miRNA-182-5p (miRNA-MUT-bio) or NC-bio through Lipofectamine reagent following the manufacturer's recommendations (Thermo Fisher Scientifc). $48 \mathrm{~h}$ after transfection, the cells were lysed to collect the lysates in different study group. Then the lysates were incubated with streptavidin magnetic beads. Finally. The PCR was performed for detection of the immunoprecipitated RNA.

\section{Statistical analysis}

The results from three individual experiments are analyzed via using SPSS 20.0 software (IBM, CA, USA). The final data were presented as mean \pm standard deviation. The difference comparation was performed via using one-way ANOVA. $* \mathrm{P}<0.05$ represents statistical significance.

\section{Results}

MiRNA-182-5p deficiency exerted protective effects on colitis induced by DSS in mice. Firstly, we established the experimental colitis model (Fig. 1A). Compared with control group, the miRNA-182-5p level was up-regulated in the model group (Fig. 1B), suggesting that the aberrant expression of miRNA-182-5p may be a potential therapeutic target in colitis. Therefore, we further evaluated the role of silencing miRNA-182-5p in colitis. After miRNA-182-5p inhibitor treatment, the miRNA-182-5p level in colitis was downregulated significantly. Compared with control group, the colon length was shortened significantly, indicating that the colon was severely damaged (Fig. 1C). In the mean time, as shown by the H\&E staining results, the histological structure was normal and there was no sign of inflammatory infiltration in control group (Fig. 1C). In contrast, submucosal edema, goblet cells damage and inflammation infiltration were observed in the colitis model group. In 
A

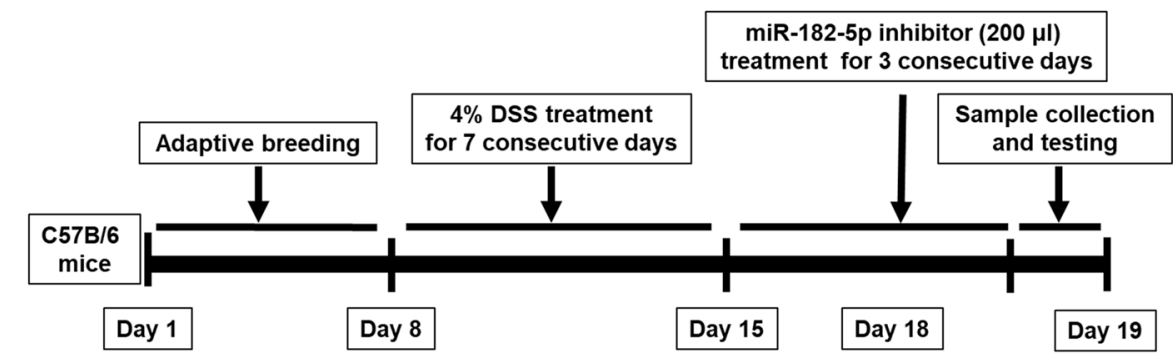

C
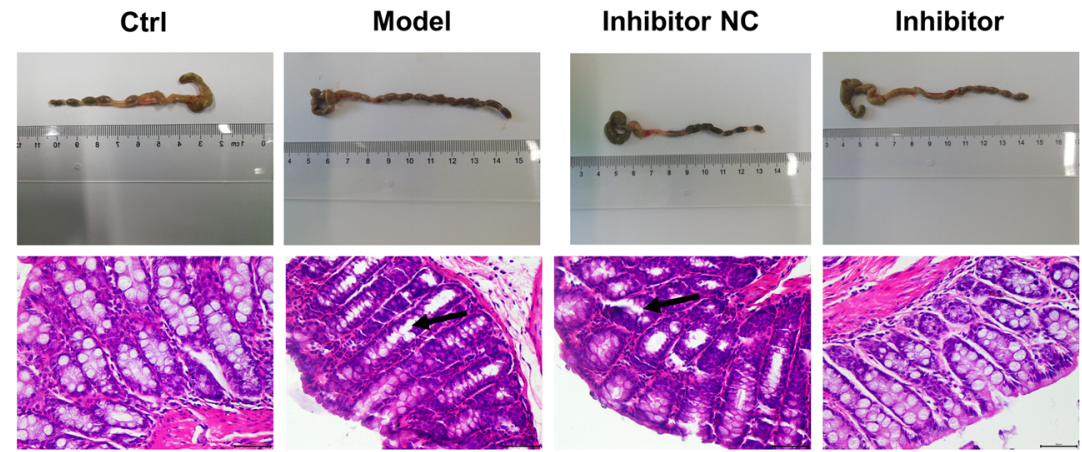

D

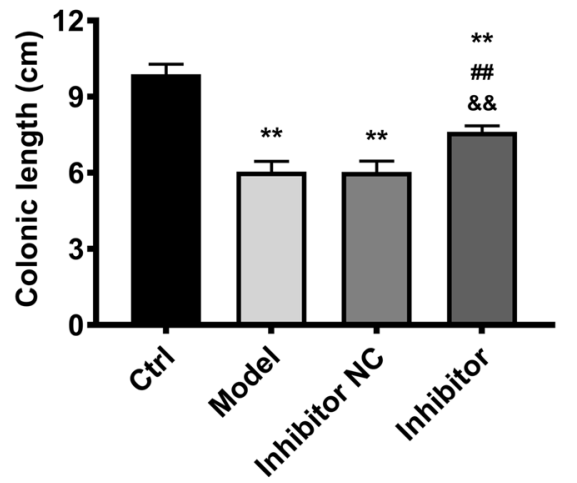

Fig. 1 Downregulation of miRNA-182-5p exerts protective effects on colitis stimulated by DSS in mice. The timeline of miRNA-182-5p effects on colitis stimulated by DSS in mice (A); The miRNA-182-5p level evaluated by PCR in the different study groups $(\mathbf{B})$; The colonic

addition, the colonic damage index was elevated evidently in model group (Fig. 1D). All these results proved that the colitis model was established successfully. Moreover, the histopathological changes, shortening of colonic length and colonic damage index were all reduced by miRNA-182-5p inhibitor, confirming that miRNA-182-5p deficiency have protective effects on colitis induced by DSS.

Inhibition of miRNA-182-5p exerted protective effects in colitis model via suppression of inflammation response as well as oxidative stress and elevation of TGF- $\beta 1$. The inflammatory factors IL-21, IL-17 A, IL-6 were up-regulated significantly in colitis model group in contrast to control, while miRNA-182-5p inhibitor alleviated the increase of IL-21, group

\section{B}
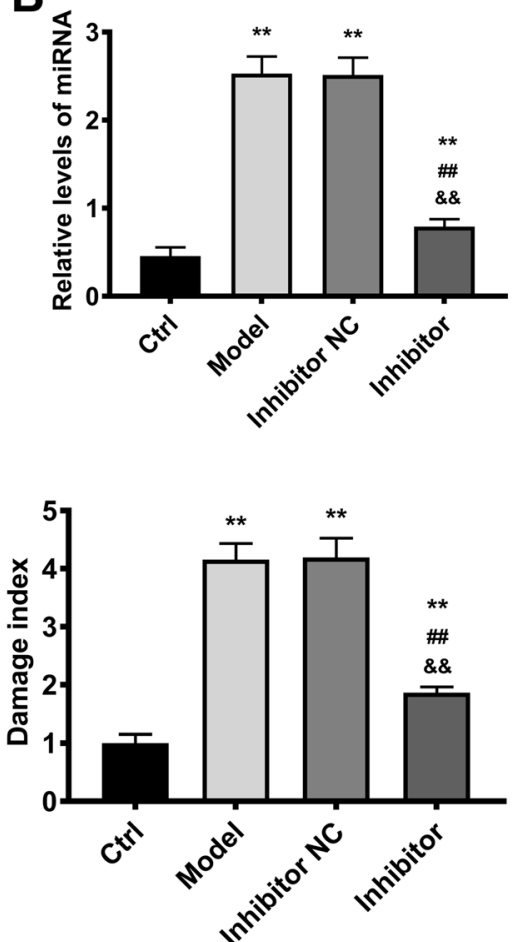

length, representative images of $H \& E$ staining and damage index in the study groups, the scales $=50 \mu \mathrm{m}(\mathbf{C}$ and $\mathbf{D}) * * \mathrm{P}<0.01 \mathrm{Vs}$. Control group; ${ }^{\# \#} \mathrm{P}<0.01 \mathrm{~V}$ s. model group; ${ }^{\&} \mathrm{P}<0.01 \mathrm{Vs}$. inhibitor $\mathrm{NC}$

IL-17 A, and IL-6 (Fig. $2 \mathrm{~A}-\mathrm{C}$ ). TGF- $\beta 1$ is a crucial wound healing factor and has promotional effect on tight junction protein expression [28]. TGF- $\beta 1$ was decreased remarkably in colitis group when compared with control. MiRNA$182-5 p$ inhibitor treatment resulted in significant increase in TGF- $\beta 1$ level as compared with model group (Fig. 2D). SOD and MDA as indicators of oxidative stress were also evaluated herein. Compared with control, the SOD was declined and MDA was increased obviously in colitis model group, suggesting that the oxidative stress was enhanced in model group, while inhibitor alleviated the decrease of SOD and the increase of MDA (Fig. 2E, F). The findings herein confirmed that miRNA-182-5p could alleviate colonic damage 

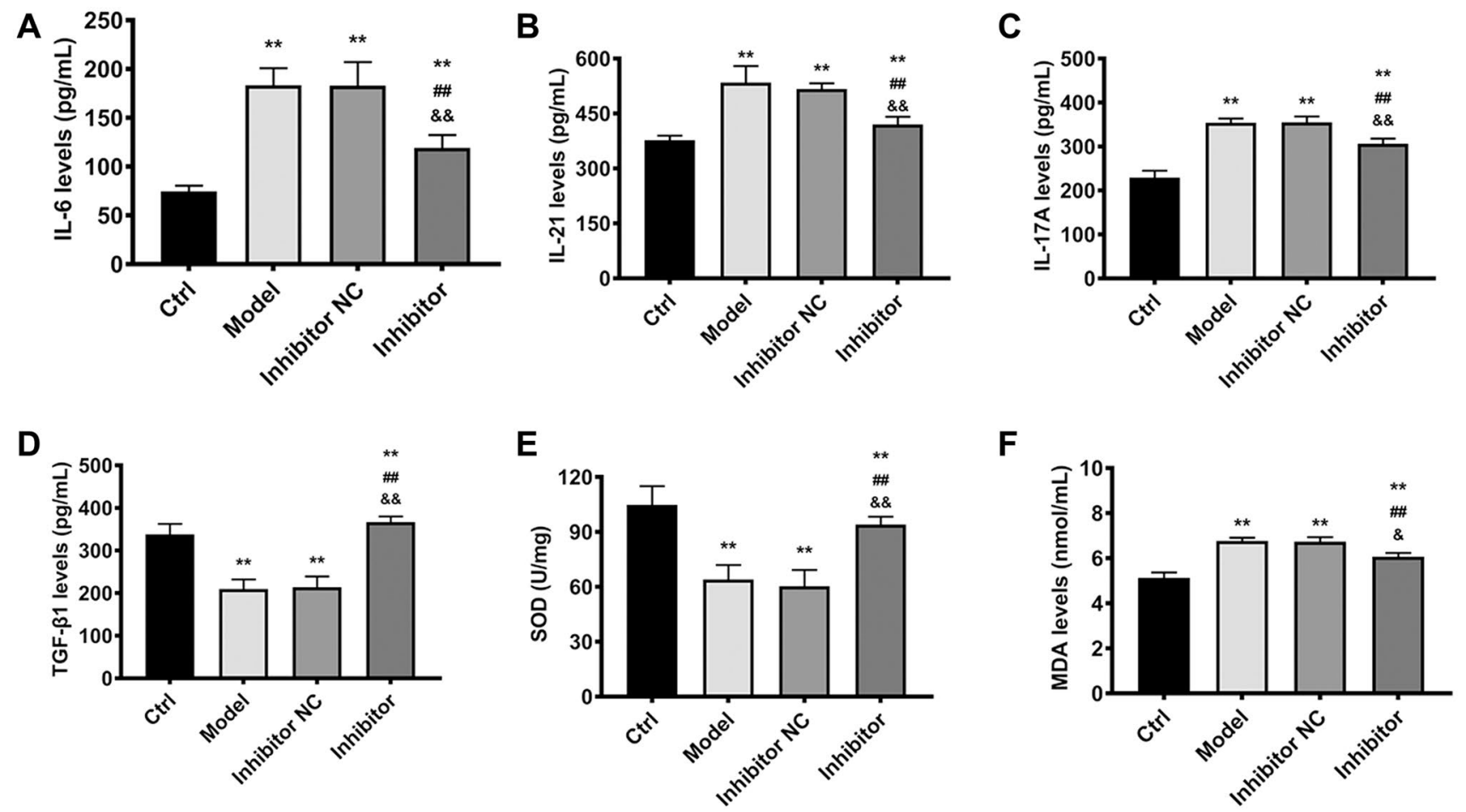

Fig. 2 The effects of miRNA-182-5p on inflammatory factors, oxidative stress and TGF- $\beta 1$ level. The inflammatory factors, oxidative stress and TGF- $\beta 1$ level in the study groups. ${ }^{*} \mathrm{P}<0.01$ Vs. Control group; ${ }^{\#} \mathrm{P}<0.01 \mathrm{Vs}$. model group; ${ }^{\& \&} \mathrm{P}<0.01 \mathrm{Vs}$. inhibitor NC group

via elevation of TGF- $\beta 1$ and suppression of inflammation response and oxidative stress.

Claudin-2 was the binding target of miRNA-182-5p and was negatively regulated by miRNA-182-5p level in colitis model. Claudin-2 was predicted to be the target of miRNA182-5p via online tool (microRNA.org) (Fig. 3A). This was further validated via luciferase reporter assay and RNA pulldown assay (Fig. 3B, C). The lowest luciferase activity and claudin-2 enrichment level was found in (WT) RNA mimic group and miRNA-bio group respectively, strongly supporting that claudin- 2 was targeted by miRNA-182-5p. The claudin-2 level was declined evidently in colitis group. As shown by PCR and immunohistochemical results, miRNA-182-5p inhibitor caused a significantly increase in claudin-2 level as compared with control (Fig. 3D, E), suggesting that the claudin-2 level was negatively regulated by miRNA-182-5p.

Silencing miRNA-182-5p alleviated colonic damage via targeting claudin- 2 . As claudin- 2 was confirmed as the target of miRNA-182-5p, the following experiments was performed to determine whether miRNA-182-5p functions via targeting claudin-2. If miRNA-182-5p functions via targeting claudin-2, the effects of miRNA-182-5p inhibitor will be in accordance with claudin- 2 overexpression. Consistent with the results above, claudin- 2 was reduced significantly in colitis model and overexpressed evidently in claudin-2 overexpression group (Fig. 4A, B). The colonic length, histopathological image and colonic damage index (Fig. 4C, D) in control or model group were consistent with the results in Fig. 1 as described above. Claudin- 2 overexpression resulted in improvement in the shortening of colonic length, the histopathological changes and colonic damage index, which was consistent with the effects of miRNA-182-5p inhibitor in colitis model. The effects of claudin-2 overexpression on inflammatory factors, oxidative stress and TGF- $\beta 1$ were also in line with the effects of miRNA-182-5p inhibitor (Fig. 5). The increase of IL-6, IL-21, IL-17 A and MDA were partially inhibited by claudin- 2 overexpression. While the decrease of TGF- $\beta 1$ and SOD were partially inhibited by claudin-2 overexpression. All the findings suggested that silencing miRNA-182-5p protected against colonic injuries via targeting claudin- 2 .

\section{Discussion}

Inflammatory bowel disease is one of the major chronic digestive system diseases with features of increasing incidence and threatening patients' quality of live [7]. The gut epithelium is the crucial barrier against pathogen invasion. The epithelial barrier impairment has been confirmed as the main etiological factor for colitis [29-32]. Increasing studies have showed that miRNAs have played a vital role in the 
A
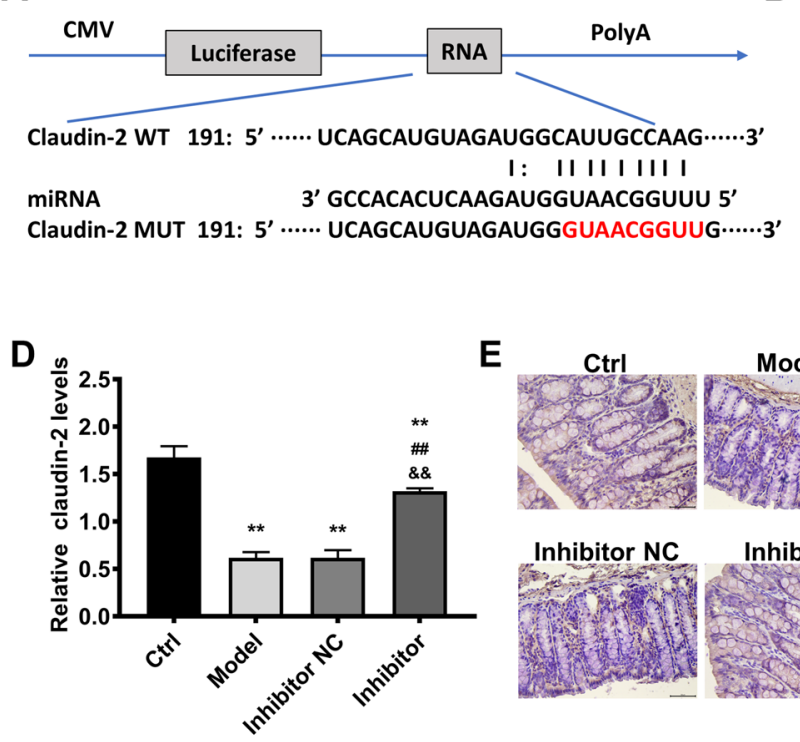

E
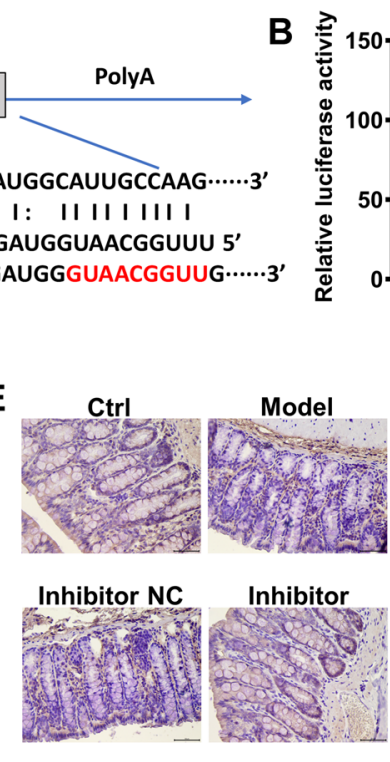
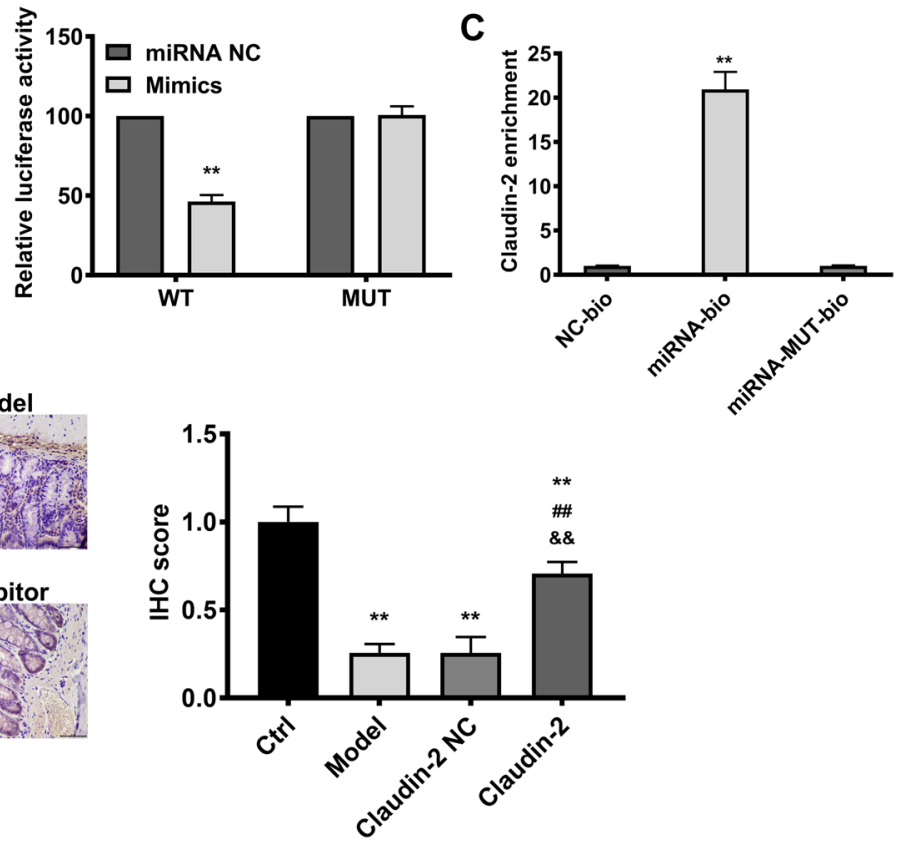

Fig. 3 Predication and validation of the target of miRNA-182-5p. The target binding sites predicted via microRNA.org online tool (A) and validated via luciferase reporter assay and RNA pull-down assay

regulation of epithelial barrier function. Intestinal epithelial barrier function can be enhanced via miR-381-3p knockdown [33]. MiR-24 is reported to act as a crucial regulator in intestinal barrier [34]. MiR-429 overexpression can cause intestinal barrier dysfunction [35]. Therefore, it's meaningful to find the aberrantly expressed miRNA which acts as a vital regulator in the onset and progress of colitis.

Firstly, we established the colitis model via using DSS. Consistent with colitis model in the literatures, the colonic length was shortened evidently and the damage index was elevated [36-38]. In addition, as seen from the H\&E staining, submucosal edema, goblet cells damage and inflammation infiltration occurred in the colitis model which is also consistent with previous reports $[37,38]$.

MiRNA-182-5p is a new emerging miRNA and has been confirmed to be involved in several diseases [23, 39-42]. In this study, miRNA-182-5p was found to be up-regulated significantly in colitis model group, which indicates an underlying role of it in colitis. We speculate that inhibition of miRNA-182-5p may help to alleviate ulcerative colitis We conjectured that inhibition of miRNA-182-5p will alleviate experimental ulcerative colitis. Just as expected, the colonic length, damage index and pathological lesion were all improved in miRNA-182-5p inhibitor group when compared with control group. We found that the miRNA-182-5p is a promising therapeutic target in colitis treatment.

Inflammation response and oxidative stress are closely associated with the initiation and pathogenesis of colitis.
(B and C); The Claudin-2 levelsin the study groups, the scales $=50$ $\mu \mathrm{m}(\mathrm{D}$ and $\mathrm{E}) . * * \mathrm{P}<0.01$ Vs. Control group; \#\#P<0.01Vs. model group; \&\&P $<0.01 \mathrm{Vs}$. inhibitor NC group

The inflammation response is the eternal theme in colitis therapy. The inflammatory level is elevated in colitis and excessive generation of inflammatory cytokines causes colon injuries and contributes to the progress of colitis [43, 44]. Consistent with the previous literatures, both inflammatory factors and oxidative stress level were increased in model group [43, 44]. In this study, the inflammatory indicators including IL-21, IL-17 A, IL-6 were all declined in miRNA$182-5 p$ inhibitor group when compared with model group. Our findings are largely consistent with a recent report in which miRNA-182-5p attenuates inflammation in cerebral ischemia-reperfusion injury[45]. These data suggested that inhibition of miRNA-182-5p is a promising strategy for inflammation control in colitis. It is known to all that oxidative stress is caused by disequilibrium between oxidative system and anti-oxidative system. In this research, the antioxidant indicator, SOD in model group was increased by miRNA-182-5p inhibitor. While the peroxidation product MDA, induced by DSS, was deceased by miRNA-182-5p inhibitor. Consistent with these findings, miR-182-5p inhibited oxidative stress and apoptosis triggered by oxidized lowdensity lipoprotein via targeting toll-like receptor 4[46].In addition, TGF- $\beta 1$, which is generated by inflammatory and non-inflammatory cells, is vital immune reactions suppressor [47]. In this research, we found that the TGF- $\beta 1$ level inhibited by DSS was elevated by miRNA-182-5p inhibitor. As discussed above, results support that downregulation of miRNA-182-5p has therapeutic effects in colitis. 


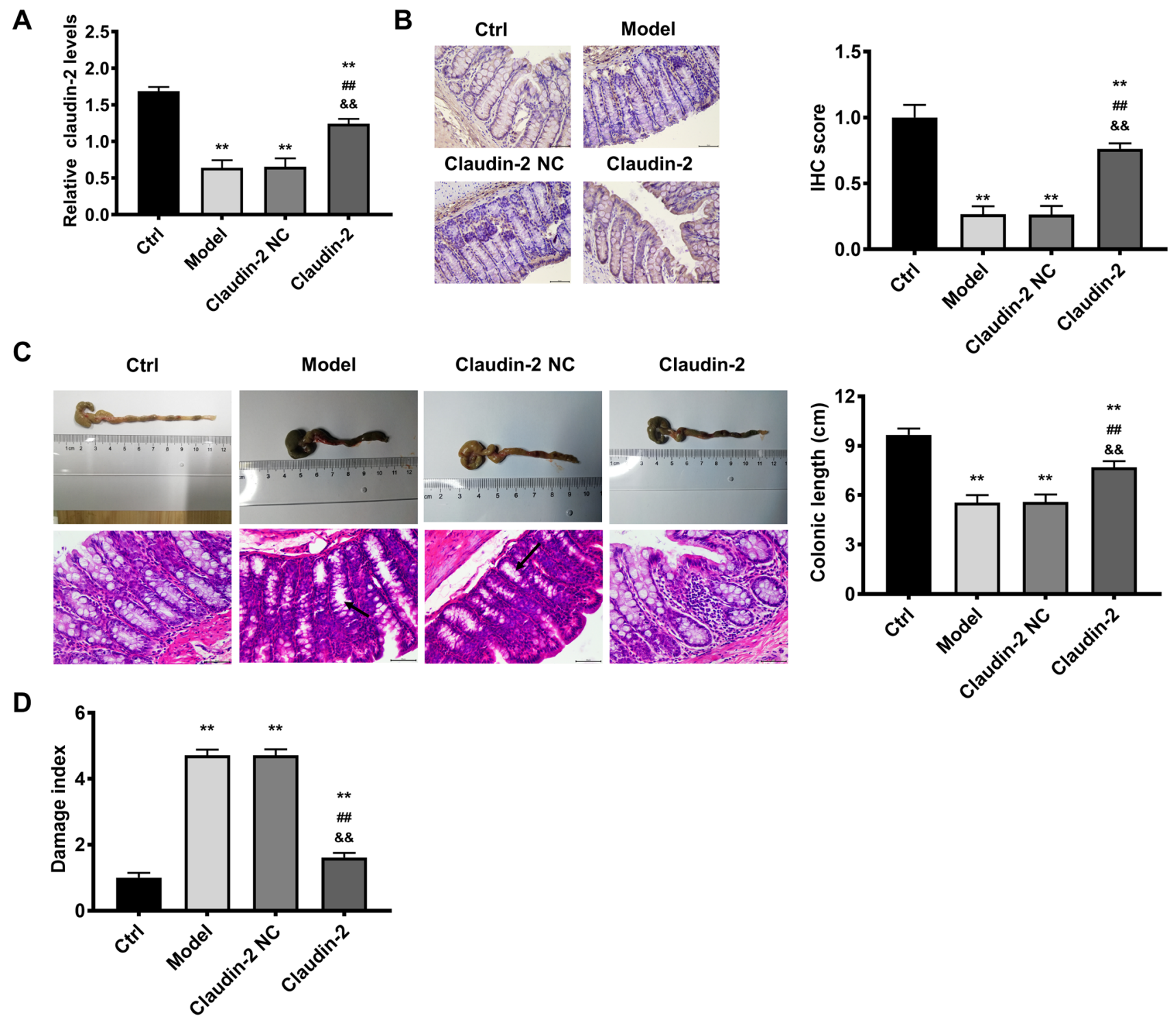

Fig. 4 Claudin-2 overexpression exerts protective effects on colitis induced by DSS in mice. Claudin-2 levels in the study groups (A, B); The colonic length, representative images of H\&E stain-

Emerging evidence supports the notion that miRNA can complement and bind to target genes and directly cut mRNA, so as to regulate the level of target mRNA[48, 49]. The underlying mechanism for the role of miRNA-182-5p was further examined herein. We found that claudin-2 mRNA was targeted by miRNA-182-5p. Claudin- 2 is one of the tight proteins which play pivotal roles in maintaining intestinal mucosal permeability. The tight junction proteins are essential in maintaining integrity of intestinal barrier. Dysfunction of intestinal barrier results in rectal bleeding and diarrhea, contributing to the onset and development of colitis [50]. In the present study, the decrease of claudin-2 was found in model group. After inhibition of miRNA-182-5p, ing and damage index in the study groups, the scales $=50 \mu \mathrm{m}(\mathrm{C}$ and $\mathrm{D})$. ${ }^{* *} \mathrm{P}<0.01$ Vs. Control group; ${ }^{\# \#} \mathrm{P}<0.01 \mathrm{Vs}$. model group; $\& \& \mathrm{P}<0.01$ Vs. Claudin-2 NC group

claudin-2 was up-regulated evidently. This indicated that miRNA-182-5p functions in colitis via targeting claudin-2 and regulating claudin- 2 expression.

The effects of claudin- 2 overexpression were investigated in colitis to determine whether inhibition of miRNA-182-5p functions via upregulation of claudin-2. The pathological changes, colonic length, damage index in model group and TGF- $\beta 1$ levels were all found to be improved by claudin- 2 overexpression, which was consistent with the results in miRNA-182-5p inhibitor group. Such observation is in fact consistent with previous studies [51, 52]. Claudin- 2 overexpression also exerted anti-inflammation and anti-oxidative stress effects. Collectively, all the effects of miRNA-182-5p 

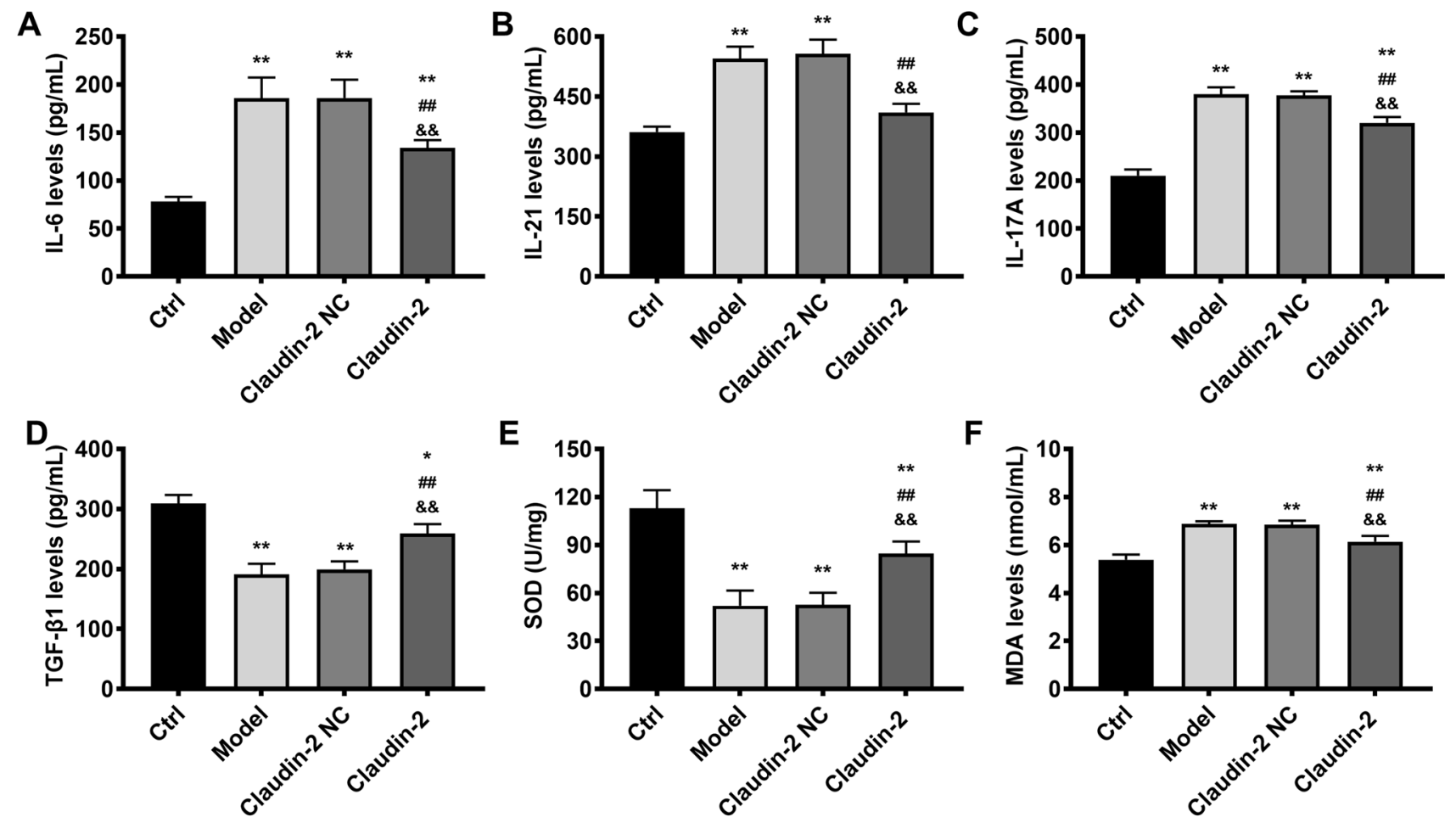

Fig. 5 The effects of claudin- 2 overexpression on inflammatory factors, oxidative stress and TGF- $\beta 1$ level. The inflammatory factors, oxidative stress and TGF- $\beta 1$ level in the study groups. ${ }^{* *} \mathrm{P}<0.01 \mathrm{Vs}$. Control group; ${ }^{\# \#} \mathrm{P}<0.01 \mathrm{Vs}$. model group; ${ }^{\&} \mathrm{P}<0.01 \mathrm{Vs}$. Claudin-2 NC group

inhibitor in colitis can be achieved via claudin-2 overexpression. Claudin-2 overexpression was also found in miRNA182-5p inhibitor group, strongly elucidating that inhibition of miRNA-182-5p exerts protective effects in colitis via targeting and up-regulating claudin-2 expression.

\section{Conclusions}

In the present study, miRNA-182-5p was overexpressed in colitis model and inhibition of miRNA-182-5p alleviated experimental ulcerative colitis induced by DSS via targeting claudin-2 in vivo. The effects included anti-inflammation, anti-oxidation, up-regulation of TGF- $\beta 1$ and improvement of pathological damages. While the effects of miRNA-182-5p inhibition in colitis is still needed to be validated in clinic.

Funding There was no funding received for this study.

\section{Declarations}

Conflict of interest The authors declare that they have no conflict of interest.

Ethical approval This study was approved by the Ethics Committee of the Shenzhen Traditional Chinese Medicine Anorectal Hospital.
Informed consent Written informed consent for publication was obtained from each participant.

Open Access This article is licensed under a Creative Commons Attribution 4.0 International License, which permits use, sharing, adaptation, distribution and reproduction in any medium or format, as long as you give appropriate credit to the original author(s) and the source, provide a link to the Creative Commons licence, and indicate if changes were made. The images or other third party material in this article are included in the article's Creative Commons licence, unless indicated otherwise in a credit line to the material. If material is not included in the article's Creative Commons licence and your intended use is not permitted by statutory regulation or exceeds the permitted use, you will need to obtain permission directly from the copyright holder. To view a copy of this licence, visit http://creativecommons.org/licenses/by/4.0/.

\section{References}

1. Matsuoka K, Kobayashi T, Ueno F, Matsui T, Hirai F, Inoue N, Kato J, Kobayashi K, Kobayashi K, Koganei K, Kunisaki R, Motoya S, Nagahori M, Nakase H, Omata F, Saruta M, Watanabe T, Tanaka T, Kanai T, Noguchi Y, Takahashi KI, Watanabe K, Hibi T, Suzuki Y, Watanabe M (2018) Sugano K and Shimosegawa T. Evidence-based clinical practice guidelines for inflammatory bowel disease. J Gastroenterol 53:305-353

2. Bernstein CN, Fried M, Krabshuis JH, Cohen H, Eliakim R, Fedail S, Gearry R, Goh KL, Hamid S, Khan AG, LeMair AW, Malfertheiner, Ouyang Q, Rey JF, Sood A, Steinwurz F, Thomsen 
OO, Thomson A, Watermeyer G (2010) World gastroenterology organization practice guidelines for the diagnosis and management of IBD in 2010. Inflamm Bowel Dis 16:112-124

3. Chachu KA, Osterman MT (2016) How to diagnose and treat IBD mimics in the refractory IBD patient who does not have IBD. Inflamm Bowel Dis 22:1262-1274

4. Shivashankar R, Lichtenstein GR (2018) Mimics of inflammatory bowel disease. Inflamm Bowel Dis 24:2315-2321

5. Ng WK, Wong SH, Ng SC (2016) Changing epidemiological trends of inflammatory bowel disease in Asia. Intest Res 14:111-119

6. Chi KR (2016) Epidemiology: rising in the east. Nature 540:S100-S102

7. Molodecky NA, Soon IS, Rabi DM, Ghali WA, Ferris M, Chernoff G, Benchimol EI, Panaccione R, Ghosh S, Barkema HW, Kaplan GG (2012) Increasing incidence and prevalence of the inflammatory bowel diseases with time, based on systematic review. Gastroenterology 142:46-54.e42; quiz e30

8. Terzić J, Grivennikov S, Karin E, Karin M (2010) Inflammation and colon cancer. Gastroenterology 138:2101-2114.e5

9. Abraham BP, Ahmed T, Ali T (2017) Inflammatory bowel disease: pathophysiology and current therapeutic approaches, in gastrointestinal pharmacology. In: B Greenwood-Van Meerveld (ed)., Springer International Publishing, Cham

10. D'Haens GR, Sartor RB, Silverberg MS, Petersson J, Rutgeerts P (2014) Future directions in inflammatory bowel disease management. J Crohns Colitis 8:726-734

11. Actis GC, Pellicano R, Fagoonee S, Ribaldone DG (2019) History of inflammatory bowel diseases. J Clin Med 8

12. Chandan K, Gupta M, Sarwat M (2019) Role of host and pathogen-derived microRNAs in immune regulation during infectious and inflammatory diseases. Front Immunol 10:3081

13. Hoefel G, Tay H, Foster P (2019) MicroRNAs in lung diseases chest 156:991-1000

14. Scalavino V, Liso M, Serino G (2020) Role of microRNAs in the regulation of dendritic cell generation and function. Int $\mathrm{J}$ Mol Sci. 21

15. Schulte LN, Bertrams W, Stielow C, Schmeck B (2019) ncRNAs in inflammatory and infectious diseases. Methods Mol Biol 1912:3-32

16. Shi Y, Dai S, Qiu C, Wang T, Zhou Y, Xue C, Yao J, Xu Y (2020) MicroRNA-219a-5p suppresses intestinal inflammation through inhibiting Th1/Th17-mediated immune responses in inflammatory bowel disease. Mucosal Immunol 13:303-312

17. Ge Y, Sun M, Wu W, Ma C, Zhang C, He C, Li J, Cong Y, Zhang D, Liu Z (2019) MicroRNA-125a suppresses intestinal mucosal inflammation through targeting ETS-1 in patients with inflammatory bowel diseases. J Autoimmun 101:109-120

18. Zimmerman NP, Vongsa RA, Wendt MK, Dwinell MB (2008) Chemokines and chemokine receptors in mucosal homeostasis at the intestinal epithelial barrier in inflammatory bowel disease. Inflamm Bowel Dis 14:1000-1011

19. Martens EC, Neumann M, Desai MS (2018) Interactions of commensal and pathogenic microorganisms with the intestinal mucosal barrier. Nat Rev Microbiol 16:457-470

20. Yin J, Zhou C, yang K, Ren Y, Qiu Y, Xu P, Xiao W, Yang H. Mutual regulation between butyrate and HIF- $1 \alpha$ in epithelial cell promotes expression of tight junction (TJ) proteins. Cell Biol Int

21. Sharma D, Malik A, Guy CS, Karki R, Vogel P, Kanneganti TD (2018) Pyrin Inflammasome Regulates Tight Junction Integrity to Restrict Colitis and Tumorigenesis. Gastroenterology 154:948964.e8

22. Guo Y, Wu X, Wu Q, Lu Y, Shi J, Chen X (2018) Dihydrotanshinone I, a natural product, ameliorates DSS-induced experimental ulcerative colitis in mice. Toxicol Appl Pharmacol 344:35-45
23. Fang YN, Huang ZL, Li H, Tan WB, Zhang QG, Wang L, Wu JL (2018) Highly expressed miR-182-5p can promote preeclampsia progression by degrading RND3 and inhibiting HTR-8/SVneo cell invasion. Eur Rev Med Pharmacol Sci 22:6583-6590

24. Yang J, Liu XX, Fan H, Tang Q, Shou ZX, Zuo DM, Zou Z, Xu M, Chen QY, Peng Y, Deng SJ, Liu YJ (2015) Extracellular vesicles derived from bone marrow mesenchymal stem cells protect against experimental colitis via attenuatingcolon inflammation, oxidative stress and apoptosis. PLoS One 10:e0140551

25. Amirshahrokhi K (2019) Febuxostat attenuates ulcerative colitis by the inhibition of NF- $\mathrm{KB}$, proinflammatory cytokines, and oxidative stress in mice. Int Immunopharmacol 76:105884

26. Liang Y, Song X, Li Y, Chen B, Zhao W, Wang L, Zhang H, Liu Y, Han D, Zhang N, Ma T, Wang Y, Ye F, Luo D, Li X, Yang Q (2020) LncRNA BCRT1 promotes breast cancer progression by targeting miR-1303/PTBP3 axis. Mol Cancer 19:85

27. Gu P, Chen X, Xie R, Xie W, Huang L, Dong W, Han J, Liu X, Shen J, Huang J, Lin T (2019) A novel AR translational regulator IncRNA LBCS inhibits castration resistance of prostate cancer. Mol Cancer 18:109

28. Troncone E, Marafini I, Stolfi C, Monteleone G (2018) Transforming growth factor- $\beta 1 / \mathrm{Smad} 7$ in intestinal immunity, inflammation, and cancer. Frontiers in immunology 9:1407-1407

29. Sun X, Yang Q, Rogers CJ, Du M, Zhu M-J (2017) AMPK improves gut epithelial differentiation and barrier function via regulating Cdx2 expression. Cell death differentiation 24:819-831

30. Huang S, Fu Y, Xu B, Liu C, Wang Q, Luo S, Nong F, Wang X, Huang S, Chen J, Zhou L, Luo X (2020) Wogonoside alleviates colitis by improving intestinal epithelial barrier function via the MLCK/pMLC2 pathway. Phytomedicine 68:153179

31. Katinios G, Casado-Bedmar M, Walter SA, Vicario M, GonzalezCastro AM, Bednarska O, Soderholm JD, Hjortswang H, Keita AV (2020) Increased colonic epithelial permeability and mucosaleosinophilia in ulcerative colitis in remission compared with irritable bowelsyndrome and health. Inflamm Bowel Dis

32. Tao Y, Yue M, Lv C, Yun X, Qiao S, Fang Y, Wei Z, Xia Y, Dai Y (2020) Pharmacological activation of ERbeta by arctigenin maintains the integrity of intestinal epithelial barrier in inflammatory bowel diseases. Faseb j 34:3069-3090

33. Liu L, Yao J, Li Z, Zu G, Feng D, Li Y, Qasim W, Zhang S, Li T, Zeng H, Tian X (2018) miR-381-3p knockdown improves intestinal epithelial proliferation and barrier function after intestinal ischemia/reperfusion injury by targeting nurr1. Cell Death Dis 9:411

34. Soroosh A, Rankin CR, Polytarchou C, Lokhandwala ZA, Patel A, Chang L, Pothoulakis C, Iliopoulos D, Padua DM (2019) miR24 is elevated in ulcerative colitis patients andregulates intestinal epithelial barrier function. Am J Pathol 189:1763-1774

35. Yu T, Lu XJ, Li JY, Shan TD, Huang CZ, Ouyang H, Yang HS, Xu JH, Zhong W, Xia ZS, Chen QK (2016) Overexpression of miR429 impairs intestinal barrier function in diabetic mice by downregulating occludin expression. Cell Tissue Res 366:341-352

36. de Paula do Nascimento R, Lima AV, Oyama LM, Paiotti APR, Cardili L, Martinez CAR, Pereira JA, Silva MF, Garofolo IC, Silveira VLF, Caperuto LC (2020) Extra virgin olive oil and flaxseed oil have no preventive effects on DSS-induced acute ulcerative colitis. Nutrition. 74:110731

37. Rabah H, do Carmo FLR, Carvalho RDO, Cordeiro BF, da Silva SH, Oliveira ER, Lemos L, Cara DC, Faria AMC, Garric G, Harel-Oger M, Le Loir Y, Azevedo V, Bouguen G, Jan G (2020) Beneficial propionibacteria within a probiotic emmental cheese: impact on dextran sodium sulphate-induced colitis in mice. Microorganisms. 8

38. Din AU, Hassan A, Zhu Y, Zhang K, Wang Y, Li T, Wang Y, Wang G (2020) Inhibitory effect of Bifidobacterium bifidum 
ATCC 29521 on colitis and its mechanism. J Nutr Biochem 79:108353

39. Fang X, Sun R, Hu Y, Wang H, Guo Y, Yang B, Pi J, Xu Y (2018) miRNA-182-5p, via HIF2alpha, contributes to arsenic carcinogenesis: evidence from human renal epithelial cells. Metallomics 10:1607-1617

40. Hirata H, Ueno K, Shahryari V, Tanaka Y, Tabatabai ZL, Hinoda Y, Dahiya R (2012) Oncogenic miRNA-182-5p targets Smad4 and RECK in human bladder cancer. PLoS One 7:e51056

41. Li Y, Li L (2019) Prognostic values and prospective pathway signaling of MicroRNA-182 in ovarian cancer: a study based on gene expression omnibus (GEO) and bioinformatics analysis. J Ovarian Res 12:106

42. Yang L, Dou Y, Sui Z, Cheng H, Liu X, Wang Q, Gao P, Qu Y, Xu M (2020) Upregulated miRNA-182-5p expression in tumor tissue and peripheral blood samples from patients with non-small cell lung cancer is associated with downregulated Caspase 2 expression. Exp Ther Med 19:603-610

43. Gerges SH, Tolba MF, Elsherbiny DA, El-Demerdash E (2020) The natural flavonoid galangin ameliorates dextran sulphate sodium-induced ulcerative colitis in mice: effect on Toll-like receptor 4 , inflammation and oxidative stress. Basic Clin Pharmacol Toxicol

44. Rapa SF, Waltenberger B, Di Paola R, Adesso S, Siracusa R, Peritore AF, D’Amico R, Autore G, Cuzzocrea S, Stuppner H, Marzocco S (2020) Plumericin prevents intestinal inflammation and oxidative stress in vitro and in vivo. Faseb j 34:1576-1590

45. Wang J, Xu Z, Chen X, Li Y, Chen C, Wang C, Zhu J, Wang Z, Chen W, Xiao Z, Xu R (2018) MicroRNA-182-5p attenuates cerebral ischemia-reperfusion injury by targeting Toll-like receptor 4. Biochem Biophys Res Commun 505:677-684

46. Qin SB, Peng DY, Lu JM, Ke ZP (2018) MiR-182-5p inhibited oxidative stress and apoptosis triggered by oxidized low-density lipoprotein via targeting toll-like receptor 4. J Cell Physiol 233:6630-6637

47. Troncone E, Marafini I, Stolfi C, Monteleone G (2018) Transforming Growth Factor-beta1/Smad7 in Intestinal Immunity, Inflammation, and Cancer. Front Immunol 9:1407

48. Li ZN, Ge MX, Yuan ZF (2020) MicroRNA-182-5p protects human lens epithelial cells against oxidative stress-induced apoptosis by inhibiting NOX4 and p38 MAPK signalling. BMC Ophthalmol 20:233

49. Jiang L, Zhang M, Wang S, Xiao Y, Wu J, Zhou Y, Fang X (2020) LINC01018 and SMIM25 sponged miR-182-5p in endometriosis revealed by the ceRNA network construction. Int J Immunopathol Pharmacol 34:2058738420976309

50. Jeon Y-D, Lee J-H, Lee Y-M, Kim D-K (2020) Puerarin inhibits inflammation and oxidative stress in dextran sulfate sodiuminduced colitis mice model. Biomed Pharmacother 124:109847

51. Weber CR, Nalle SC, Tretiakova M, Rubin DT, Turner JR (2008) Claudin-1 and claudin-2 expression is elevated in inflammatory bowel disease and may contribute to early neoplastic transformation. Lab Invest 88:1110-1120

52. Raju P, Shashikanth N, Tsai PY, Pongkorpsakol P, Chanez-Paredes S, Steinhagen PR, Kuo WT, Singh G, Tsukita S, Turner JR (2020) Inactivation of paracellular cation-selective claudin-2 channels attenuates immune-mediated experimental colitis in mice. J Clin Invest 130:5197-5208

Publisher's Note Springer Nature remains neutral with regard to jurisdictional claims in published maps and institutional affiliations. 